\title{
The Analysis on Social Justice and Its Impact Factors-- Based on the Analysis of CGSS Questionnaire Data
}

\author{
Yajie Han $^{1, \text { a }}$ Yali Liu ${ }^{2, \text { b }}$ Lei Zhou ${ }^{3, \mathrm{c}}$ \\ ${ }^{1,2,3}$ Beijing Normal University, Beijing, China, 100875 \\ ${ }^{\mathrm{a} e m a i l,}{ }^{\mathrm{b}}$ email, ${ }^{\mathrm{c} e m a i l}$
}

Keywords: Social Justice, Factor Analysis, Multiple Linear Regression, Hysteresis Characteristics

\begin{abstract}
Social justice is a person of fairness subjective judgment, with the continuous development and progress of society, people's demands for a fair society continues to strengthen. To study the sense of fairness and social factors In this paper, China General Social Survey (CGSS) data. Firstly, a clear sense of social justice measure. Secondly, the data for each of China's General Social Survey conducted a preliminary analysis and processing, using one-way ANOVA determine whether an explanatory variable has a significant impact on social justice, no significant effect of excluding variable. Then, the rest of the variables for factor analysis, pick out the greatest explanatory power factor, then multiple linear regression on all the factors and social justice using the least squares method to give each of the main factors for social justice impact the size of the . The 10 - 13 years of questionnaires have done more to give four years and the main factors combined to give a greater degree of influence of the nine main factors; different gender, education, age group, we get a questionnaire classification stepwise regression different groups in society a sense of fairness factors; by the time of horizontal plane and the vertical plane of analysis, preliminary exploration of these factors changes with time.

In addition, the paper came up with one of the geographical factor, given by the data, we built a model of the time a sense of fairness and social and economic development trends are analyzed, concluded: lateral view, at a certain time node regional economic level and local residents a sense of fairness and social development is a negative correlation; longitudinal view, real GDP per capita growth in all regions over time, and social justice in all regions showing the changes over time of uncertainty, and we also found in some areas, a sense of fairness with respect to the pace of economic development has a certain lag.
\end{abstract}

\section{Introduction}

Discuss social issues of fairness that long. In the American psychologist John • Si Taxi • Adams (John Stacy Adams) equity theory, people judge fairness, that sense of fairness, tend to come from social comparison. Social justice is an objective phenomenon and researchers can go to study from all angles and to develop criteria to measure the objective reality.

But man for fairness judgment is a subjective feeling, does not comply with the fixed criteria, all different, it is difficult to find a suitable thing to measure such a subjective experience.

In real life, a direct impact on human behavior is not fair degree of attitude, but the public perception of fairness. Social justice part of the impact of one's own life and mentality, but there is a certain influence on the development of society. Feel a fair comparison in society, often show a positive attitude, such high confidence, willing to spend, etc., which for the prosperity of society, there are certain advantages; feel injustice in social comparison of people, often exhibit problems emotional instability or a crisis of confidence, go a long time will cause great harm to the social stability. Therefore, the study of social justice is not only a personal psychological problems help, but also to government policy, adjustment system has a significant role.

We therefore based Chinese General Social Survey (CGSS) questionnaire data, discussion and analysis, through data analysis and modeling, solve the following two questions:

Analysis the main factors those affecting the sense of fairness of civil society and the variation of these factors. 
Create a time model depicts the relationship between social justice and economic development trends of the population.

\section{The Analysis on Problem}

In this paper, 2010--2013 questionnaire in both the metric as a topic, namely "Overall, do you think today's society is fair?" This metric is a value of 1, 2, 5 of discrete ordinal variables. Because this question is the attitude of the public sense of fairness most direct reflection of society, from the viewpoint of accuracy and convenience should choose this as a measure of justice indicators. But it is worth noting that, in 2005 and 2006. This year's survey, we did not find that mentioned above having such a clear point of subjective evaluation questions. And the question is present in a given six-year data both appeared. However, taking into account the objectives of this question as a measure of social justice will have a sample selection bias problem that only work and have income residents will head to answer this question, for those residents unemployed or without income, their social Justice has not been reflected, so we decided to put this question only as a 05 and 06 of the measure, and only for problem analysis II. And through access to information, we know 05--06 years of data and 10 - 13 years of data belonging to different project period, there may be a big difference on the issue of setting.

One problem requires analysis of the main factors affecting people's sense of social justice, and the variation of these factors can affect different groups of factors from the point of view is the same. Horizontal analysis focuses on many factors and the extent of the impact of social sense of fairness. The main problem is in the screening division of a lot of factors, and the regression equation.

Second problem need to establish a time models characterize the relationship between social justice and economic development trends of the population. Key is a longitudinal analysis of the relationship between social justice and economic development trends. The main problem is defined in the sense of fairness and social and economic trends, as well as methods of longitudinal analysis.

\section{The Question Assumes}

Questionnaire information is accurate and reliable, and universal.

Classification questionnaire is not issued, that there is no significant sample selection bias problem.

\section{The Model}

The Establishment of Single Factor Variance Analysis Model. For each year questionnaires were analyzed, pick out a certain number of explanatory variables, but we still cannot determine whether a correlation between all of the explanatory variables and the dependent variable is social justice, therefore, the first step, we need to based on the one-way ANOVA did on many variable filter, removing no significant impact on the sense of fairness of social variables, to simplify the subsequent analysis. Our age, income and other continuous variables according to certain criteria classified, making it into a categorical variable, such as age into the old, the young, the income is divided into high, medium and low. SPSS software using one-way ANOVA analysis results were as follows (in 11 years of data, for example)

A total of 43 explanatory variables, according to the significance can be judged, gender, ethnic and other 11 variables and social justice little correlation cannot be considered.

Evaluation on the Society Fairness Impact Factors Based on Factor Analysis. According to the results of SPSS-way ANOVA analysis, the current total of 33 selected high correlation variables. However, ANOVA only prove a factor in whether the impact on social justice, cannot be further determine how the degree of influence of each variable on social justice, and the number of single-factor analysis of complex extraction of social justice indicators of each other there may be between the problem, it can reduce the dimension principal component factor analysis, to avoid 
problems. First, we need to use SPSS software to determine whether the data suitable for factor analysis. Bartlett spherical test is designed to test whether the correlation matrix for the matrix, if so, that the factor model is inappropriate. In general, the level of significance value $(<0.05)$ smaller, then the more likely there is a relationship between the original variables, if the significance level is greater than 0.05 , then the data is not suitable for factor analysis. In this problem, 11 years of data calculated by SPSS, a significance of .000 , it is suitable for factor analysis.

KMO test: The higher the value KMO measure (close to 1.0), indicating that the common factor between the variables, the more data is suitable for factor analysis. Generally, KMO $>0.9$ is very good, as good 0.8-0.9, 0.7-0.8 general, the difference was 0.6 or less. Combination of these two indicators, 11 years of data suitable for factor analysis, additional years of data are also suitable for factor analysis.

Table.1 KMO and Bartlett Test

\begin{tabular}{|cc|c|}
\hline \multicolumn{2}{|c|}{ Kaiser-Meyer-Olkin } & .764 \\
Bartlett & Chi-square & 32749.322 \\
& Df & 780 \\
& Significance & .000 \\
\hline
\end{tabular}

Extract the main ingredient. Select the characteristic value based on the principle of values $>1=$ factor most initial factor. As the table shows, the first 10 factors with values greater than 1 , and can explain the dependent variable proportion reached $62.942 \%$, which can explain most of the changes in the social sense of fairness, the selection of these 10 as the host molecule.

Rotation factor. Rotation the factor loading matrix, so that the structure factor loading matrix is simplified. The maximum variance rotation method, the following table describes the factor matrix after rotation, according to this matrix, the sub-factor into the respective main factor.

Table.2 Rotating element matrix

\begin{tabular}{|c|c|c|c|c|c|c|c|c|c|c|}
\hline 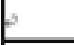 & 10 & 20 & 30 & 4. & 50 & 60 & 70 & 80 & 90 & $10 \%$ \\
\hline 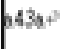 & $.949-$ & $?$ & & o & & & b & & & \\
\hline 4356 & .949 & & & & & & & & & \\
\hline 4300 & $.826-$ & & & & & & $-182 \nmid$ & & & \\
\hline aseo & .798 & $.139+$ & & & -.11 & & & & & \\
\hline 432. &.$\infty$ & $.2360^{\circ}$ & & & & $.358+$ & & & & \\
\hline 390 & & .728 & -1389 & 1994 & & .252. & $.144 \uparrow$ & & & \\
\hline 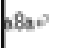 & .173 & 6897 & & 120. & $\cdot, 10^{\circ}$ & & $=100$ & & & \\
\hline 272 & & $.669\}^{\circ}$ & & .174 & & .304. & -229 & & & \\
\hline 250 & & $-.647 \mathrm{f}^{\circ}$ & & $-.181-$ & & & -.186 & & & -106 \\
\hline$=50$ & & $-.573 \uparrow$ & & -.4334 & & $-.116+$ & & .131 & & \\
\hline 2750 & .188 & $.554+$ & & .166 & & 163. & $=.249$. & & & $=103$. \\
\hline 10 & & $.516 t^{\circ}$ & & & & -152 & .262 & .226. & & .242 \\
\hline 41 & & $-496-$ & & .2004 & & -182 & -245 & & $=.155$ & . 191. \\
\hline $3230 \times 4$ & & & .004 & & & & & & & \\
\hline 3254 & & & .000 & & & & & & & \\
\hline 33000 & & & $.855+$ & & & & & & & \\
\hline 180 & & $.262\}^{\circ}$ & & .8004 & & & .103 & & & \\
\hline 2740 & & $.273 \dagger^{\circ}$ & & .060 ' & & & & & & \\
\hline
\end{tabular}

Factor score. When each primary factor can get a reasonable explanation, may be regarded as the main factor is a linear combination of the original variables in order to obtain the main factor scores derived value that is the main factor, the factor score coefficient matrix: 
Table.3 Component score coefficient matrix

\begin{tabular}{|c|c|c|c|c|c|c|c|c|c|c|}
\hline 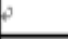 & 10 & 2 & 30 & 4 & 50 & 6 & 7 & 8 & 90 & 10 \\
\hline 541 & .028 & -.227 & -.016 & .278 & .021 & $=.075+$ & -.217 & .040 & $-.120+$ & .174 \\
\hline $85 a^{2}$ & .024 & -.122 & $\cdot .017$ & -.171 & 011. & 039 & $\cdot, 021$ & 089 & .001 & .041 \\
\hline 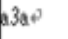 & .032 & $=.008$ & .001 & .040 & $\cdot .009$ & $=.060+$ & .459 & $=.027$ & .039 & -.001 \\
\hline 25 & .033 & -.053 & -.031 & .022 & -.082 & .027 & -.013 & .222 & .721 & .044 \\
\hline $27 a$ & $=.037$ & .242 & $\cdot .015$ & $=.065$ & .033. & .022 & $\cdot .077$ & .042 & $=.021$ & 039 \\
\hline $8 a+$ & .008 & .271 & .046 & -.066 & .006 & -.134 & -.076 & .008 & $=.020$ & $\cdot .006$ \\
\hline 10 & -017 & .273 & .041 & -.135 & .043. & -.152 & .131 & .162 & .037 & .222 \\
\hline 18 & .000 & -.102 & .014 & .498 & .000 & -.005 & .016 & .033 & $.013+$ & .012 \\
\hline $32 \mathrm{ca}$ & 007 & .023 & .3601 & .004 & -.014 & .009 & .029 & -.015 & -.021 & -030 \\
\hline $\mathrm{a} 2 \mathrm{cb}$ & $\cdot .003$ & .039 & .386 & .015 & -.010 & .003 & .016 & .038 & $=.030+$ & .028 \\
\hline $32 \mathrm{cc}$ & -.001 & .028 & .383 & .022 & $-.008+$ & -.0024 & .000 & .044 & $.008+$ & .014 \\
\hline 2330 & $=.049$ & .031 & .020 & $=.028$ & .028 & .0534 & .039. & .570 & -.040 & $=.027$ \\
\hline a 340 & .000 & .023 & -.020 & $=.048$ & -.027 & $=.0014$ & $.086+$ & -.569 & $-.125+$ & $.07 n$ \\
\hline $243 a^{2}$ & .296 & -.024 & .005 & .001 & .038 & -.028 & .055 & $\cdot .019$ & $.028+$ & $=.007$ \\
\hline 4360 & .296 & $=.024$ & .005 & .001 & .038 & $=.008+$ & $.055^{4}$ & $\cdot .019$ & $.028+$ & $=.007$ \\
\hline
\end{tabular}

Each year, more than step data are processed to obtain the primary factor for each year, then four years of the primary factors combined to give 4-year deal with some of the main common factor:

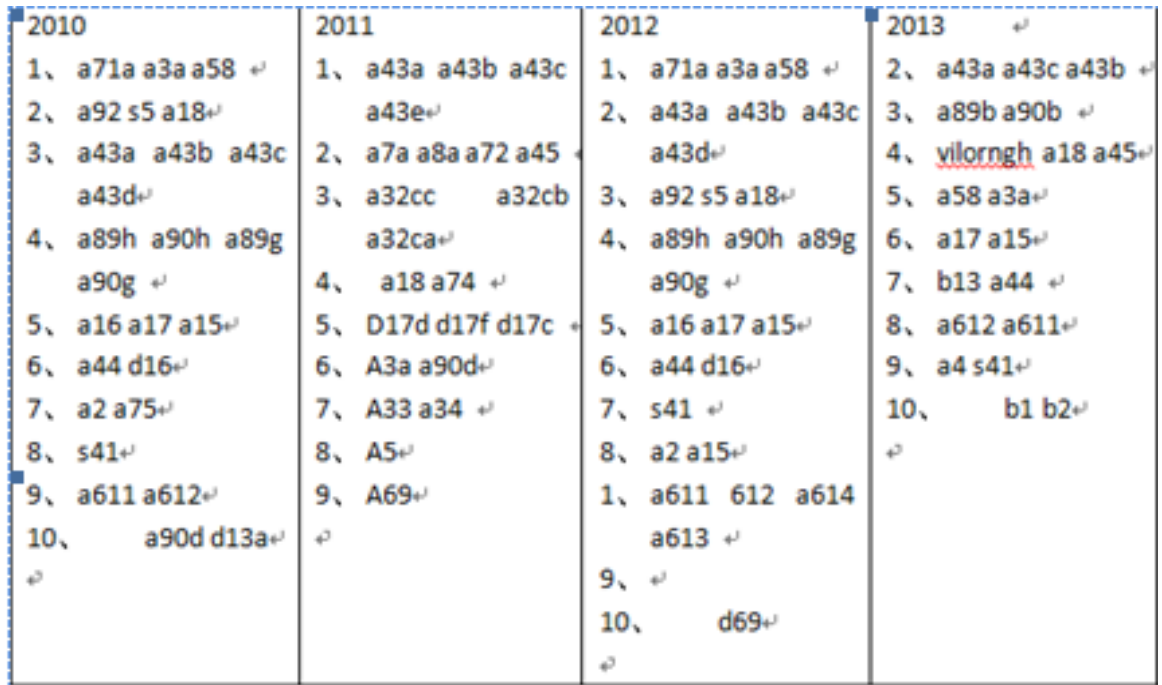

And the main factor is named

\begin{tabular}{|c|c|}
\hline Factor1 & $\begin{array}{c}\text { Ratings flow factor (past and present, present and future of } \\
\text { contrast) }\end{array}$ \\
\hline Factor2 & Life experience factors (age, work experience, etc.) \\
\hline Factor3 & Family factor (parents working condition) \\
\hline Factor4 & Mental health factors (effort, irritability degree) \\
\hline Factor5 & Social factors (social insurance, social experience, etc.) \\
\hline Factor6 & Geographical factors (regional, national, etc.) \\
\hline Factor7 & Political factors (to vote, union membership, etc.) \\
\hline Factor8 & Economic factors (socio-economic status, income, etc.) \\
\hline Factor9 & Health factors (physical health) \\
\hline
\end{tabular}


Model validation. In 11 years of data, for example, according to the residual histogram shows that the residuals approximately normal distribution with mean 0 , there is no obvious, but goodness of fit adjusted R2 of 0.287 small, the impact of factors Social justice is not fully included in these explanatory variables.

Using the Least Squares Regression Method to Analysis the Main Factors Affecting Social Justice. Independent sample T-test, test different gender, education, human education is a key factor of social justice.

We can see that in the larger grade mobility, social factors and other aspects of social justice between men and women affect the intensity, relatively speaking and the impact of social factors on women's significant impact on the perceived level of remarkable men.

We can see that the health factors had no significant effect on the sense of fairness of people with different educational society have an impact in other ways.

We can see that, for the elderly, life experience factor, the social factor is their sense of fairness society is very important factor for the middle-aged in terms of economic factors, political factors, grade liquidity factor is their sense of fairness society is very important influencing factors for young people in terms of family factors, geographical factor is their sense of fairness society is very important factor.

If you explore these factors as changes of time, we can cross perspective and people of different ages can be seen as a timeline of changes in factors of people of all ages a sense of social justice can be seen as these factors along with time variation. With longitudinally extending time of each factor on the impact of social justice efforts is changing.

You can also view from the vertical plane, with the economic impact of the passage of time, social progress, revenue, accounts and other geographical factors on the sense of fairness is gradually reduced, if voted, whether suffered unfair treatment and other political, social influence factors gradually improved.

The Relations between the Sub-Regional Social Justice Average and the Per Capita Real GDP. Because too few periods of GDP, to establish the time series model sample periods is not enough, so we use the plot points plotted ways to build time model, look at the relationship between economic development and civil society with a sense of fairness difference method.

We use the same prices as well as the total area population statistics yearbook, 2005 as the base year, calculated 05 - real per capita GDP 13-year sub-region - in 2006, and 10. We first social area residents a sense of fairness mean longitudinal axis to a logarithmic per capita real GDP is the horizontal axis, respectively, made the 2010--2013 years scatter plot. Corresponding correlation coefficients were highly significant:

Table 4 The correlation coefficient and its significance

\begin{tabular}{ccc}
\hline Year & Coefficient & Significance (p) \\
\hline 2010 & -0.328 & 0.0717 \\
2011 & -0.1947 & 0.3404 \\
2012 & -0.3298 & 0.0806 \\
2013 & -0.3124 & 0.1056
\end{tabular}

The Relations between the Sub-Region Social Justice and the Mean Per Capita Real GDP Growth Rate. We used the interpolation model to fill the 07--09 years 3 years vacant value of GDP and the use of social justice mean 05 and 06 years still interpolation model predicted a sense of fairness in the middle three years. We describe the relationship between the 28 provincial-level administrative units of social justice and real GDP per capita growth rate between provincial levels.

From the figure we can see that the average per capita relative social justice in terms of real GDP growth in Henan Province has obvious lag effect. And the right sense of fairness, Jiangsu Province, the average social relative per capita real GDP growth rate in terms of what the law is not, the trend mess. Perhaps this different circumstances and the degree of economic development in the region 
related to this article but unfortunately did not do further analysis and testing.

\section{Conclusion}

The lateral view, on (such as a year) one time node, the level of economic and social development of local residents a sense of fairness is a negative correlation between the lateral view, real GDP per capita growth in all regions over time, while the sub-region's social Justice is showing changes over time of uncertainty, and we also found that, in most areas, the sense of fairness with respect to the pace of economic development has a certain lag.

\section{Model Improvement}

The estimated model parameters factor analysis after using structural equation modeling to its improvement. The model has validation features, it can test the reasonableness of factor analysis, and can be a good analysis of the resulting correction factor parameters, increase legitimacy and credibility of the analysis results.

Research on the relationship between justice and socio-economic trends, especially in areas of social justice may be found through chart with respect to GDP growth with a certain lag, and this lag may be related to the degree of economic development of this model can also conduct in-depth analysis and testing.

\section{References}

[1] Liu Junqiang. Economic Growth, Vol. 6 (2004) No 53, p.25-26

[2] Zhao Xiaohang. Educational Research, Vol. 12 (2005) No 27, p.74-76

[3] Wang Yijie. Economic and Technological Cooperation, Vol. 30 (2004) No 19, p.144-145

[4] Hu Jianguo. Economic Growth, Vol. 29 (2008) No 27, p.21-23

邮寄地址：北京市海淀区新街口外大街 19 号

收件人：韩亚洁 18811795260 